\title{
Health seeking behaviors among Syrian refugee women: A scoping review
}

\author{
Areej Al-Hamad*1, Cheryl Forchuk², Abe Oudshoorn², Gerald McKinley ${ }^{3}$ \\ ${ }^{1}$ School of Nursing, University of Calgary in Qatar, Qatar \\ ${ }^{2}$ Arthur Labatt Family School of Nursing, Western University, London, Ontario, Canada \\ ${ }^{3}$ Schulich School of Medicine \& Dentistry, Western University, London, Ontario, Canada
}

Received: April 7, 2020

DOI: $10.5430 /$ ijh.v6n2p54
Accepted: May 29, 2020

Online Published: June 10, 2020

\begin{abstract}
Objective: The aim of this scoping review was to explore what is known about the health seeking behaviors among Syrian refugee women within hospital and community settings. The objectives were to explore health seeking behaviors of Syrian refugee women and highlight specific issues that are important to Syrian refugee women related to seeking for health care. In addition, to uncover potential obstacles/barriers that face Syrian refugee women while seeking for health care.

Methods: Arksey and O'Malley (2005) framework for scoping review was utilized to guide the review process and to explore health seeking behaviors of Syrian refugee women. Various databases such as, Academic Search Complete, CINAHL, PubMed, Scopus, PsycINFO, Science direct and SocINDEX were utilized in the review. The search strategy consisted of broad queries, in the title, abstract, full texts and keyword fields. The search included studies published from 2011 on, that is, from the time the Syrian conflict started (March 2011) and were limited to those in the English language. Thematic analysis was undertaken to synthesize extracted data.

Results: A total of 19 studies were selected for this review. The data from this review were organized along two thematic categories (accessibility of health care services) and (awareness of health care needs and available health care services). Accessibility of health care services was the most frequently reported theme throughout this area of the literature and is most often include (financial barriers, language barriers, availability of culturally and gender appropriate health services). Awareness of health care needs and available health care services was the second commonly encountered theme within the area of Syrian refugee's women health seeking behaviors.

Conclusions: Having available and accessible health care services for Syrian refugee women in host countries is a fundamental human right that can enhance Syrian refugee women health, wellbeing and quality of life. An awareness of the potential health seeking behaviors, obstacles/barriers and challenges that facing Syrian refugee women may help to promote their accessibility to health services and consequently improve their health and wellbeing. Practitioners can use this review findings to inform future policy, education and developments in practice. Further research is required to develop and evaluate initiatives that target the identified barriers and help Syrian refugee women to fulfil their unmet health care needs.
\end{abstract}

Key Words: Health, Health seeking behaviors, Syrian refugee women, Scoping review

*Correspondence: Areej Al-Hamad; Email: areej.alhamad@ucalgary.ca; Address: School of Nursing, University of Calgary in Qatar, Qatar. 


\section{INTRODUCTION}

Syrian refugees expose to various stressors, challenges and prevalence of common consequences of exposure to trauma and high stress. ${ }^{[1]}$ Due to growing challenges and burden of health care needs among both Syrian refugees and host communities, an evidence-based recommendation to address these burden and needs are crucial. ${ }^{[2]}$ In particular, refugee women may encounter various challenges in accessing healthcare services in their host communities because of the fear of uncertainty, language barriers, cultural differences, and economic problems. $^{[3]}$ For instance, Tayfur, Günaydin ${ }^{[4]}$ conclude that one of the major concerns for countries hosting refugees from Syria is the ability to accommodate the health care needs of Syrian refugee. Another deficiency in refugee health care has been the availability of preventative services for infectious diseases. ${ }^{[5]}$ The aim of the current review is to explore what is known about the health seeking behaviors among Syrian refugee women and what are the potential obstacles that face Syrian refugee women while seeking for health care. Therefore, this review will offer opportunities for multidisciplinary collaboration to develop relevant insights and potential responses to the health seeking behaviors among Syrian refugee women and ultimately the impact on their health. Arksey and O'Malley ${ }^{[6]}$ framework was used to exploring and recognizing health seeking behaviors among Syrian refugee women. This framework aims to map the existing research but does not address the issue of quality of studies. ${ }^{[6]}$ Utilizing such a holistic framework, cannot only enhance our knowledge and understanding of the Syrian refugee women's needs and concerns, but also help empowering and fulfilling their needs and promoting their health and wellbeing. In order to confirm that no other reviews existed about health seeking behaviors among Syrian refugee women, an exploration of the literature was conducted. An extensive search of various data bases did not find any current or planned reviews on this topic.

\subsection{Review question/objective}

The primary research question that guided this study was: what has been published with regards to health seeking behaviors among Syrian refugee women? more specifically, what is the current evidence with regards to health seeking behaviors and the potential obstacles that face Syrian refugee women while seeking for health care? This research question will lead to the identification of gaps and opportunities in the existing knowledge of health seeking behaviors among Syrian refugee women. The objectives of the present study were to:

(1) Explore health seeking behaviors of Syrian refugee women.

Published by Sciedu Press
(2) Highlight specific issues that are important to Syrian refugee women related to seeking for health care.

(3) Uncover potential obstacles/barriers that face Syrian refugee women while seeking for health care.

\subsection{Phenomena of interest}

This scoping review exploring health seeking behaviors of Syrian refugee women over the age of 18 years who been forcibly displaced or migrate from their home country. For the purpose of this review, health-seeking behaviors of Syrian refugee women defined as a "any action or inaction undertaken by individuals who perceive themselves to have a health problem or to be ill for the purpose of finding an appropriate remedy."[7] Syrian refugee woman was defined as a "Syrian citizen who, owing to ongoing war in Syria, was compelled to leave his place of habitual residence in order to seek refuge."[2] The review highlights pertinent issues and concerns face Syrian refugee women while they are seeking for healthcare in host countries and identifies specific needs in relation to their health and wellbeing. Also, obstacles and barriers that Syrian refugee women encountered while they are seeking for health care have been elucidated. This review considers studies that have been conducted among Syrian refugee women in hospital or community settings.

\section{Methods}

The scoping review has become a popular approach for knowledge synthesis of research evidence that allow for mapping broad topics. ${ }^{[8]}$ Dowling, Enticott ${ }^{[9]}$ argued that scoping reviews are rigorous and using methods that allow for replication, however, it lacks the step of synthesizing or aggregating the findings to the extent customary in systematic reviews. This scoping review is based on Arksey and O'Malley $^{[6]}$ framework. This framework summarizes the evidence available on a topic in order to convey the breadth and depth of that topic. Moreover, this framework consists of five iterative steps: (1) identifying the research question; (2) identifying relevant studies; (3) selecting relevant studies; (4) data charting and finally; (5) collating, summarizing, and reporting the results. ${ }^{[6]}$ It is worth noted, that scoping reviews unlike systematic reviews provide an overview of the research activity rather than evaluating its quality. In another word, the methodology used in scoping reviews is more flexible that allowing the researcher to include grey literature as well as studies with diverse study designs. ${ }^{[6,8]}$

\subsection{Stage 1: Identifying the research question}

Our research question was: what has been published with regards to health seeking behaviors among Syrian refugee 
women? More specifically, the present review aims to address the following objectives:

(1) Explore health seeking behaviors of Syrian refugee women.

(2) Highlight specific issues that are important to Syrian refugee women related to seeking for health care.

(3) Uncover potential obstacles that face Syrian refugee women while seeking for health care.

\subsection{Stage 2: Identifying the relevant studies}

A comprehensive search strategy to identify relevant literature was conducted and involved searching of various electronic databases such as Academic Search Complete, CINAHL, PubMed, Scopus, PsycINFO, Science direct and SocINDEX were utilized in the review. Only English language papers published from 2011 on, that is, from the time the Syrian conflict started (March 2011) were considered in this review. With the help of professional Liberian, a double strand search strategy was applied running the thesauri terms first and then keywords. These two searches were then combined using the OR operator. Search terms used independently and then in combination included: women, health and wellbeing, Syrian refugee, and health seeking behaviors (see Table 1).

Table 1. Search terms

\begin{tabular}{llll}
\hline Health seeking behaviors & Syrian refugee & Women & Health and wellbeing \\
\hline "health seeking behavior*” & Syria* N2 refugee (EBSCO databases) & & \\
"health-seeking behavior*” & Syria* ADJ2 refugee (Ovid databases) & (Women OR Female* & Health and wellbeing \\
$\begin{array}{l}\text { (Utilize* OR access*) AND } \\
\begin{array}{l}\text { (healthcare OR “health care” OR } \\
\text { "medical care” OR "health service*”) }\end{array}\end{array}$ & Syria* AND refugee* & OR Woman) & \\
\hline
\end{tabular}

This method was repeated for each selected concept (health and wellbeing, refugee, women, and Health seeking behaviors) at the end these four different concepts were combined together using AND: Concept 1 AND Concept 2 AND Concept 3 AND Concept 4 were combined to yield the results. This strategy was initially created within Scopus, and then adapted for all other databases searched using keywords and database-specific subject headings where applicable.

\section{Inclusion \& exclusion criteria}

Inclusion criteria for this review were: (a) studies involving Syrian refugee women aged over 18 years who have been forcibly displaced or migrate from their home country, (b) articles written in English language, (c) the main topic of the document was Syrian refugee women's health seeking behaviors in hospitals or community settings, regardless of their location, (d) the selected documents had to be a journal article, conference paper, book chapter, short paper, literature review, report, or case study, and (e) the document had to be published from 2011 on, that is, from the time the Syrian conflict started (March 2011). A document was excluded from the study if it was published before 2011 or was targeting different refugee population in a language other than English.

\subsection{Stage 3: Selecting studies}

The selection process was most closely aligned with the research question. This was then followed by an analysis of the keywords contained in the title, abstract, the index terms used to describe the articles retrieved during the search and the review of full texts for the selected studies. The reviewers only included studies published from 2011 on, that is, from the time the Syrian conflict started (March 2011) and were limited to those in English. The search for unpublished or gray literature included ProQuest Dissertations and Theses and relevant key was included. The full text versions of eligible documents were retrieved and screened followed by a hand search and review of reference lists of all included studies for any additional relevant studies. The initial search retrieved 306 articles. All references retrieved from the database were added to reference management software (Endnote 9.2). Exclusion of duplicates reduced this number to 192 and a further 133 were excluded if inclusion criteria were not met in title or abstract. A review of the full text resulted in 59 studies. The selection process resulted in 18 relevant items that most closely aligned with research question and one more study was added after the hand search of reference lists of the selected studies to end up with a total of 19 studies involved in this scoping review. See Figure 1 for the selection flow diagram.

\subsection{Stage 4. Charting the data}

The fourth stage of the scoping review is charting the data which involves extracting data using a "narrative review" method to presents contextual or process-oriented information from each item included in the review. ${ }^{[6]}$ The 19 articles 
were charted in a comprehensive data extraction table that using an inclusive approach so no findings of potential value to this scoping review will be missed. Thematic analysis was undertaken to synthesize extracted data. The data extraction table includes the study characteristics such as, author information, publication year, where the study took place, study aim and design, sampling and the important results or findings (see Appendix).

\subsection{Stage 5: Collating, summarizing and reporting the} results

Arksey and O'Malley ${ }^{[6]}$ clarify that the fifth stage of the scoping framework is collating, summarizing and reporting the results. A total of 19 articles were reviewed for this study. The following overview describes the two themes identified within this scoping review. The data from this review were organized along two thematic categories (accessibility of health care services) and (awareness of health care needs and available health care services).

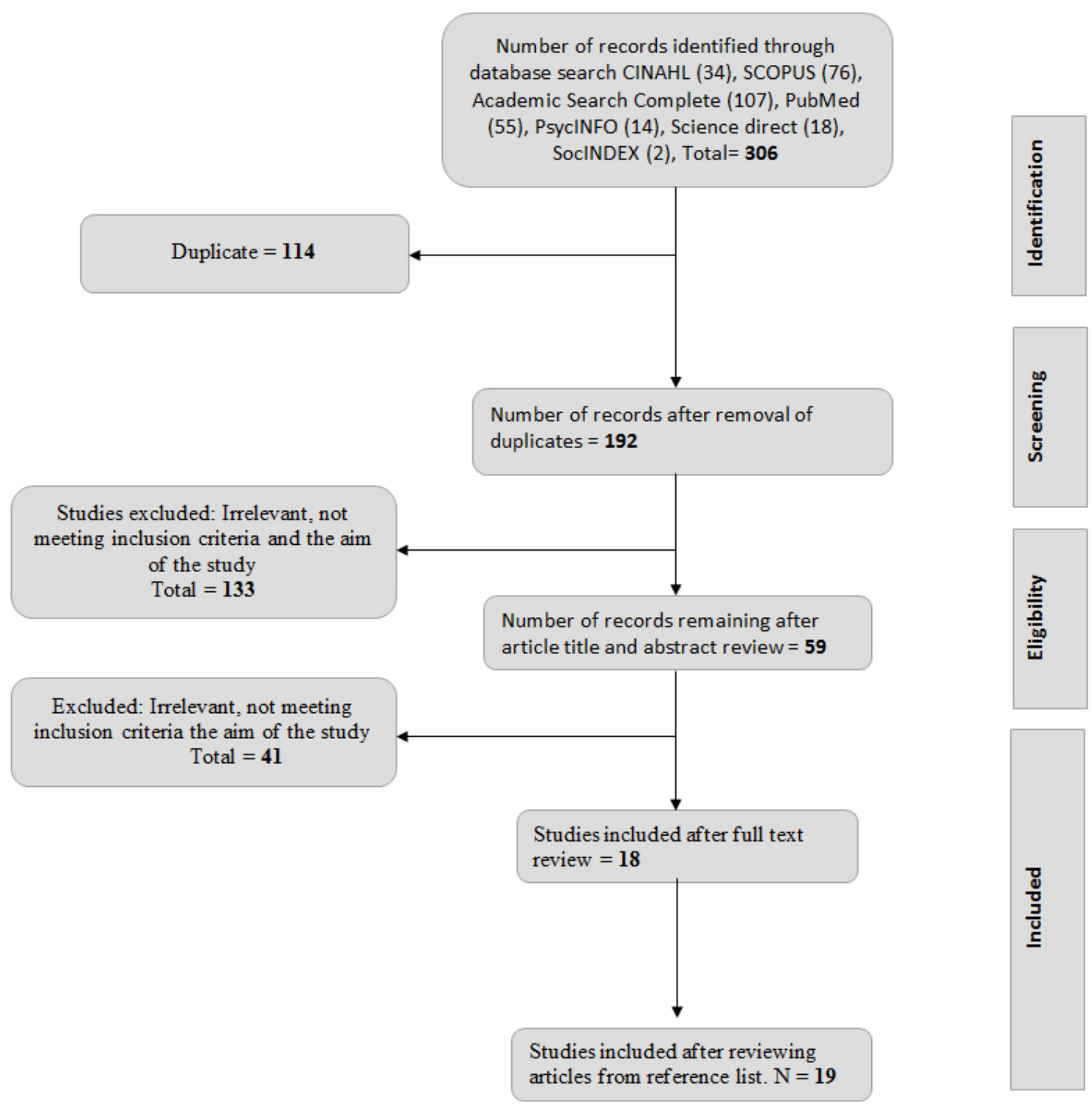

Figure 1. Selection flow diagram

\section{Results}

\subsection{Accessibility of health care services}

Given that most of the literature examines issues that affect health seeking behaviours of Syrian refugee women, accessi-

Published by Sciedu Press bility of health care services was the most frequently reported theme throughout this area of the literature and is most often include (financial barriers, language barriers, availability of culturally and gender appropriate health services). Fifteen 
of the articles selected for this scoping review reflected the financial barriers or inadequate health insurance or coverage. In general, financial barriers or the cost of services was the dominant barriers to accessing health care among Syrian refugee women. ${ }^{[5,10-18]}$

Abdin ${ }^{[18]}$ conducted a qualitative study using semi-structured interviews with 42 women in Lebanon to understand antenatal care, what it takes to treat a woman while she is pregnant and circumstances of her pregnancy in the refugee setting context. Findings revealed cost barriers of antenatal services and contraceptive cost as well as the awareness of the available antenatal services were common factors affecting antenatal care for pregnant women in the refugee setting context. Similarly, Winn, Hetherington ${ }^{[10]}$ conducted a qualitative study using semi-structured interviews with 10 health care professionals who provided care for pregnant women in Calgary, AB, to understand their experiences of caring for pregnant Syrian refugee women. The study finding's support that language, cultural and cost were the main barriers when caring for pregnant refugees. In a similar context, Guruge, Sidani, ${ }^{[16]}$ conducted a community based qualitative descriptive interpretive study that informed by Yang \& Hwang (2016) health service utilization framework. The aim of the study was to explore health care needs of newly arrived Syrian refugee women and their experiences of accessing and using the services in the Greater Toronto Area (GTA). 58 focus groups were conducted with newcomer Syrian refugee women in the GTA. The study findings revealed that socio-cultural, language, social disconnection, lack of public transportation as well as the lack of culturally and gender specific health services were common barriers for accessing health care services.

The second frequently reported barrier that affect access to health services was language barriers. ${ }^{[3,10,15,16,19-21]}$ For instance, Henry, Beruf ${ }^{[15]}$ performed a phenomenological study with 12 semi structured interview with Arabic speaking women from Syria in Germany. The aims of the study were to understand Syrian refugee women's experiences, perceived needs and expectations of health care as well as the strategies that Syrian refugee women adopt to compensate for restricted access to health care. Authors concluded that poor language skills, lack of information, cost of services and appropriate health care services were common barriers in accessing health care services among Syrian refugee women.

Similarly, Torun, Mücaz Karaaslan, ${ }^{[19]}$ conducted a mixed methods approach to assess health care needs of urban Syrian refugees' women living in Istanbul, Turkey. For the quantitative part, 891 household survey was distributed, and 31 semi-structured interviews were conducted with Syrian refugee women, key informants and Syrian NGO managers. The findings support that difficulty in accessing health care due to language barriers and lack of awareness about Turkish health care system, how to seek care for specific health care needs as well as the cost of care were the common barriers for Syrian refugee women to seek care. In a similar way, Assi, Özger-İlhan ${ }^{[21]}$ conducted literature review on Syrian refugees published between 2011 and 2019 to determine health care need and the available services to Syrian refugee women in Turkey. 122 documents were retrieved, and 13 full text articles were included in the study. The study findings revealed that language barriers and the availability of health care practitioner were the major factors that limited the effectiveness of health care services for Syrian refugees.

From availability and cultural perspective, five studies from the included studies touched based upon the availability of gender and culturally appropriate services. ${ }^{[3,10,13,15,16]}$ In particular, Erenoglu and Yaman Sozbir, ${ }^{[3]}$ conducted a randomized controlled trial with one intervention and one control group to evaluate the effect of health education given to Syrian refugee women in their language on the awareness of breast and cervical cancer in Turkey. A sample consisted of 60 Syrian refugee women (30 experiments and 30 controls). The study findings support that language, cultural and economic problems were the major contributing factors that hinder accessing health care services by Syrian refugee women. Similarly, Winn, Hetherington, ${ }^{[10]}$ concluded that cultural and language barriers including gender dynamics and wanting a female provider as well as the cost and differences in medical practices were the main barriers for pregnant refugees to navigate health care services in Canada. Moreover, Guruge, Sidani, ${ }^{[16]}$ concluded that socio-cultural factors and lack of linguistically, culturally and gender specific health services were among the common factors and barriers that affect access and use of health services by Syrian refugee women.

\subsection{Awareness of health care needs and available health care services}

Eight studies from the involved studies supported the second theme identified as knowledge and awareness of health care needs and available health care services. El Arnaout, Rutherford $^{[22]}$ conducted a systematic review to identify the primary healthcare needs of displaced Syrian in Iraq, Jordan, Lebanon, Turkey and Syria. A total of 63 articles were included in the analysis. Findings revealed that among commonly reported barriers to accessing health services were lack of awareness and education about the availability of health services and how to access these services. Similarly, Al Qadire, Aljezawi, ${ }^{[23]}$ conducted a descriptive 
cross-sectional survey design to explore the level of cancer knowledge and barriers to seeking care among Syrian refugees in Jordan. The Cancer Awareness Measure was completed by 241 Syrian refugees. The authors concluded that refugees' knowledge and awareness of available services, disease symptoms and risk factors as well as the cost of services were the common barriers to seeking care among Syrian refugees in Jordan. Another study was included in this review is a study conducted by Abdin, ${ }^{[18]}$ to understand what it takes to treat refugee women while she is pregnant in the refugee setting context. The author concluded that awareness and knowledge of available antenatal care and how to navigate and access these services was crucial to understand refugee women's pregnancy related needs and circumstances. In the same vein, Henry, Beruf ${ }^{[15]}$ conducted a phenomenological study with 12 semi-structured interviews with Arabic speaking refugee who had lived in Syria to explore Syrian refugee women experiences and the perceived needs during pregnancy. The study findings revealed that limited health literacy and lack of information around health care needs and available services were barriers in accessing health care in Germany.

Doocy, Lyles ${ }^{[17]}$ conducted a cross-sectional survey with 1,550 Syrian refugee to better understand issues related to care seeking for non-communicable disease in Jordan. The authors concluded that Syrian refugee with primary knowledge and education had the lowest rate of seeking care compared to non-educated refugee and access to medication and cost as well as provision of secondary and tertiary services were common barriers to seeking care.

An interesting cross- sectional study of 386 Syrian refugees in the GTA conducted by Oda, Hynie ${ }^{[24]}$ to assess access to health care with the perceived physical and mental health concluded that government assisted refugees had lower perceived health and access to health care compared to privately sponsored refugees due to the fact that the later had better education, socioeconomic and support system to navigate the available health services compared to government assisted refugees. A literature review conducted within the Jordanian context by El Arab and Sagbakken ${ }^{[25]}$ to identify Syrian refugee health needs and barriers to access concluded that financial and structural barriers including awareness about the availability of health services affect access to health care. Similarly, Ay González ${ }^{[26]}$ conducted a cross-sectional study with 196 survey to identify needed health care services and accessibility of Syrian refugee concluded that financial and structural barriers including the awareness of the available health services were common barriers for Syrian refugee to accessing health care services.

\section{Discussion}

This scoping review, based on 19 studies published between 2011 and on, is to our knowledge the first scoping review that include studies that have explored health seeking behaviors of Syrian refugee women. The studies were mostly conducted in the hosting countries for Syrian refugee such as Canada, Jordan, Lebanon and Turkey with a large variety in the methodological approaches that implemented in different community- and hospital settings. This scoping review highlights the need for further efforts to adopt a comprehensive approach to improve Syrian refugee women's access to health care and awareness of the available health services in their host communities.

The study findings were consistent with previous studies about health seeking behaviors of different refugee population. For instance, Marume, January ${ }^{[27]}$ conducted a study to explore if health seeking behaviors, social capital had an effect on quality of life among refugees in Zimbabwe. The authors concluded that various factors affect health seeking behaviors of refugee and their accessing to health care services such as poor economic status and psychosocial support that exist within refugee populations. Similarly, Cropley ${ }^{[28]}$ conducted a study to explore the effect of health education interventions on child malaria treatment-seeking practices among mothers in rural refugee villages in Belize, Central America. The study findings were consistent with our study in terms of the effect of knowledge and awareness of health care need and when to seeking for care. Another study that echoes our findings but in a different refugee context is a study conducted by Yun, Paul ${ }^{[29]}$ to explore barriers to care, help-seeking behaviors, and the impact of a communitybased patient navigation intervention on patient activation levels among Bhutanese refugees in the U.S. The authors concluded that financial coverage, being a bilingual and having literate family members with social networks assist on the overcome of healthcare access.

Our finding of knowledge and awareness about health needs and available health services were consistent with the study conducted by Odwe, Undie ${ }^{[30]}$ in which the authors concluded that promoting awareness and progressive attitudes towards available health care services and support or sexual and gender-based violence may encourage positive helpseeking attitudes and behaviors among Rwamwanja refugee women in Uganda.

Up to the authors' knowledge, this is the first scoping review to explore health seeking behaviors of Syrian refugee women. On the other hand, this study shares limitations that are common to scoping reviews in general, such as synthesizing different research including quantitative and qualitative 
methods in the same review while maintain balance between breadth and depth of analysis. ${ }^{[8]}$ Our passion and motivation for conducting this review was to pave the road for a more standardized and systematic evaluation of health seeking behaviours and access to care for Syrian refugee women in their hosting countries. Therefore, we aimed on identifying both breadth and depth of health seeking behaviours, barriers and challenges that Syrian refugee women encounter while seeking for health care. It is worth noted that, this review may not have captured all relevant published articles and grey literature. Searching other databases may have identified additional relevant studies, as well as relevant studies in other languages than English may have been excluded. Moreover, our definition of health seeking behaviour was broad to allow us to include a wide range of studies. Thus, we may have unintentionally excluded some studies. From a quality perspective, the quality of knowledge synthesis in this review relies on the quality of the individual studies included with an aim to capture the breadth of exploring health seeking behaviours of Syrian refugee women, rather than weighting articles in regard to method used or impact factor. ${ }^{[6,8]}$

This review provides insights that can assist researchers, health care professionals, and policymakers to understand the complexity in assessing, exploring and evaluating health seeking behaviors of Syrian refugee women in their host communities. Moreover, the findings of this review can be a basis for a more standardized and systematic evaluation. The results may also encourage stakeholder and health care professionals in planning and carrying out health care programs and services in cooperation with Syrian refugee women that are accessible, available and culturally and gender appropriate.

\section{Conclusion}

This scoping review has provided overview of studies that address health seeking behaviors for Syrian refugee women and has highlighted the barriers and challenges that those women encounter to access and navigate health care services in the host communities. A substantial evidence base supports the conclusion that Syrian refugee women face several challenges and barriers that can hinder their access to health care services and influence their health seeking behaviors in their host countries which ultimately affect their health. Accessibility of health care services as well as knowledge and awareness of health care needs and available health care services were commonly encountered themes within the area of Syrian refugee's women health seeking behaviors.

\section{CONFLiCTS OF INTEREST DisClosure}

The authors declare no conflicts of interest.

\section{REFERENCES}

[1] Javanbakht A, Amirsadri A, Suhaiban HA, et al. Prevalence of Possible Mental Disorders in Syrian Refugees Resettling in the United States Screened at Primary Care. Journal of Immigrant and Minority Health. 2019; 21(3): 664-667. PMid:30066059. https : //doi.org/10.1007/s10903-018-0797-3

[2] Naja F, Shatila H, El Koussa M, et al. Burden of non-communicable diseases among Syrian refugees: a scoping review. BMC Public Health. 2019; 19(1): 637-13. PMid:31126261. https ://doi.org/ 10.1186/s12889-019-6977-9

[3] Erenoğlu R, Yaman Sözbir Ş. The Effect of Health Education Given to Syrian Refugee Women in Their Own Language on Awareness of Breast and Cervical Cancer, in Turkey: a Randomized Controlled Trial. Journal of Cancer Education. 2020; 35(2): $241-$ 247. PMid:31414370. https://doi .org/10.1007/s13187-019 $-01604-4$

[4] Tayfur I, Günaydin M, Suner S. Healthcare service access and utilization among syrian refugees in Turkey. Annals of Global Health 2019; 85(1): 42. PMid:30896133. https://doi.org/10.5334/ aogh. 2353

[5] Doocy S, Lyles E, Roberton T, et al. Prevalence and care-seeking for chronic diseases among Syrian refugees in Jordan. BMC Public Health. 2015; 15: 1097. PMid:26521231. https ://doi .org/10.1 186/s12889-015-2429-3

[6] Arksey H, O’Malley L. Scoping studies: towards a methodological framework. International Journal of Social Research Methodology.
2005; 8(1): 19-32. https ://doi.org/10.1080/136455703200 0119616

[7] Olenja J. Health Seeking Behaviour in Context. East African Medical Journal. 2003; 80(2): 61-62. PMid:16167716. https ://doi.org/ 10.4314/eamj · v80i2.8689

[8] Pham MT, Rajić A, Greig JD, et al. A scoping review of scoping reviews: advancing the approach and enhancing the consistency. Research Synthesis Methods. 2014; 5(4): 371-385. PMid:26052958. https ://doi.org/10.1002/jrsm.1123

[9] Dowling A, Enticott J, Russell G. Measuring self-rated health status among resettled adult refugee populations to inform practice and policy-a scoping review. BMC Health Serv Res. 2017; 17(1): 817. PMid:29216897. https ://doi .org/10.1186/s12913-017 $-2771-5$

[10] Winn A, Hetherington E, Tough S. Caring for pregnant refugee women in a turbulent policy landscape: perspectives of health care professionals in Calgary, Alberta. International Journal for Equity in Health. 2018; 17(1): 91. PMid:29940958. https://doi.org/10 $.1186 / \mathrm{s} 12939-018-0801-5$

[11] Tappis H, Lyles E, Burton A, et al. Maternal health care utilization among Syrian refugees in Lebanon and Jordan. Maternal and Child Health Journal. 2017; 21(9): 1798-1807. PMid:28707099. https ://doi.org/10.1007/s10995-017-2315-y

[12] Samari G. Syrian Refugee Women's Health in Lebanon, Turkey, and Jordan and Recommendations for Improved Practice. World 
Medical and Health Policy. 2017; 9(2): 255-274. PMid:29051840. https ://doi.org/10.1002/wmh3. 231

[13] McNatt ZZ, Freels PE, Chandler H, et al. "What's happening in Syria even affects the rocks": a qualitative study of the Syrian refugee experience accessing noncommunicable disease services in Jordan. Conflict \& Health. 2019; 13: 26. PMid:31210780. https : //doi.org/10.1186/s13031-019-0209-x

[14] Lyles E, Hanquart B, Chlela L, et al. Health Service Access and Utilization among Syrian Refugees and Affected Host Communities in Lebanon. Journal of Refugee Studies. 2018; 31(1): 104-130. https://doi.org/10.1093/jrs/fex014

[15] Henry J, Beruf C, Fischer T. Access to Health Care for Pregnant Arabic-Speaking Refugee Women and Mothers in Germany. Qualitative Health Research. 2020; 30(3): 437-447. PMid:31530252. https://doi.org/10.1177/1049732319873620

[16] Guruge S, Sidani S, Illesinghe V, et al. Healthcare needs and health service utilization by Syrian refugee women in Toronto. Conflict $\&$ Health. 2018; 12: 46. PMid:30524500. https ://doi .org/10.118 6/s13031-018-0181-x

[17] Doocy S, Lyles E, Akhu-Zaheya L, et al. Health service access and utilization among Syrian refugees in Jordan. International Journal for Equity in Health. 2016; 15(1): 108. PMid:27418336. https : //doi.org/10.1186/s12939-016-0399-4

[18] Abdin L. Challenges for pregnant Syrian refugees in Lebanon. Eastern Mediterranean Health Journal. 2018; 24(10): 1026-1029. PMid:30582146. https://doi.org/10.26719/2018.24.10.10 26

[19] Torun P, Mücaz Karaaslan M, Sandıklı B, et al. Health and health care access for Syrian refugees living in İstanbul. International Journal of Public Health. 2018; 63(5): 601-608. PMid:29629476. https://doi.org/10.1007/s00038-018-1096-4

[20] Doğan N, Dikeç G, Uygun E. Syrian refugees' experiences with mental health services in Turkey: "I felt lonely because I wasn't able to speak to anyone". Perspectives in Psychiatric Care. 2019; 55(4): 673680. PMid:31093988. https://doi.org/10.1111/ppc. 12400

[21] Assi R, Özger-İlhan S, İlhan MN. Health needs and access to health care: the case of Syrian refugees in Turkey. Public Health. 2019; 172: 146-152. PMid:31235210. https ://doi.org/10.1016/j.puhe .2019 .05 .004

[22] El Arnaout N, Rutherford S, Zreik T, et al. Assessment of the health needs of Syrian refugees in Lebanon and Syria's neighbor- ing countries. Conflict and Health. 2019; 13: 31. PMid:31297141. https ://doi .org/10.1186/s13031-019-0211-3

[23] Qadire M Al, Aljezawi M, Al-Shdayfat N. Cancer Awareness and Barriers to Seeking Medical Help Among Syrian Refugees in Jordan: a Baseline Study. J Cancer Educ. 2019; 34(1): 19-25. PMid:28779440. https : //doi .org/10.1007/s13187-017-1260-1

[24] Oda A, Hynie M, Tuck A, et al. Differences in Self-Reported Health and Unmet Health Needs Between Government Assisted and Privately Sponsored Syrian Refugees: A Cross-Sectional Survey. Journal of Immigrant \& Minority Health. 2019; 21(3): 439442. PMid:29959652. https://doi.org/10.1007/s10903-018 $-0780-z$

[25] El Arab R, Sagbakken M. Healthcare services for Syrian refugees in Jordan: a systematic review. European Journal of Public Health. 2018; 28(6): 1079-1087. PMid:29905785. https ://doi.org/10 .1093/eurpub/cky103

[26] Ay M, González PA, Delgado RC. The perceived barriers of access to health care among a group of non-camp syrian refugees in Jordan. International Journal of Health Services. 2016; 46(3): 566-589. PMid:26962004. https : //doi .org/10.1177/00207314166368 31

[27] Marume A, January J, Maradzika J, et al. Social capital, healthseeking behavior and quality of life among refugees in Zimbabwe: a cross-sectional study. International Journal of Migration, Health and Social Care. 2018; 14(4): 377-386. https ://doi.org/10.1108/ I JMHSC-04-2017-0017

[28] Cropley L. The effect of health education interventions on child malaria treatment-seeking practices among mothers in rural refugee villages in Belize, Central America. Health Promotion International. 2004; 19(4): 445-452. PMid:15520038. https ://doi.org/10.1 093/heapro/dah406

[29] Yun K, Paul P, Subedi P, et al. Help-seeking behavior and health care navigation by Bhutanese refugees. J Community Health. 2016; 41(3): 526-534. PMid:26659398. https ://doi.org/10.1007/s10900 $-015-0126-\mathrm{x}$

[30] Odwe G, Undie CC, Obare F, et al. Attitudes towards help-seeking for sexual and gender-based violence in humanitarian settings: the case of Rwamwanja refugee settlement scheme in Uganda. BMC Int Health Hum Rights. 2018; 18(1): 15. PMid:29530031. https : //doi.org/10.1186/s12914-018-0154-6 